\title{
Stitch Bond Process of Pd-Coated Cu Wire: Experimental and Numerical Studies of Process Parameters and Materials
}

\author{
A. Rezvani ${ }^{1}$, M. Mayer ${ }^{1}$, I. Qin ${ }^{2}$, J. Brunner ${ }^{2}$, Bob Chylak $^{2}$ \\ ${ }^{1}$ Microjoining laboratory, University of Waterloo, Waterloo, N2L 3G1, ON, Canada \\ ${ }^{2}$ Kulicke and Soffa Industries, Inc., Fort Washington, PA 19034, USA \\ Phone: $519-888-4567$ ext 38579 \\ Email: arezvani@uwaterloo.ca
}

\begin{abstract}
Cost reduction is the main driver in the recent transition to $\mathrm{Cu}$ wire bonding from predominate $\mathrm{Au}$ wire bonding. Other cost reduction in packaging comes from new developments in substrates and lead frames, for example, Pre-Plated Frames (PPF) and uPPF for QFP and QFN reduce the plating and material cost. However, 2nd bonds (stitch bonds) can be more challenging on some of the new leadframe types due to the rough surface finish and thin plating thickness. $\mathrm{Pd}$-coated $\mathrm{Cu}(\mathrm{PCC})$ wire has been recently introduced to improve the wire bonding process with bare $\mathrm{Cu}$ wire, mainly to improve reliability and enhance the stitch bond process. More fundamental studies are required to understand the influences of bonding parameters and bonding tools to improve stitch bondability. The stitch bond process of $0.7 \mathrm{mil}$ diameter PCC wire on Au/Ni/Pd-plated quad flat-no lead (QFN) PPF substrate is investigated in this study. Two capillaries with the same geometry but different surface finishes are used to investigate the effect of capillary surface finish on the stitch bond process. The two capillary types are a polished finish type which is commonly used for Au wire bonding, and a granular finish capillary that has a much rougher surface finish. Process window between no stick on lead (NSOL) and short tail is compared. The effect of process parameters including bond force and table scrub amplitude is studied. The process window test results revealed that the granular capillary has larger process window and a lower chance of short tail occurrence. It has been shown that a higher scrub amplitude increases the chance of successful stitch bond formation. To further compare the capillary surface finishes, 3 sets of parameter settings with different bond force and scrub amplitude are tested. For all three parameter sets tested, the granular capillary showed better quality in bond strength. The granular capillary resulted in higher stitch pull strength compared to the polished type. A finite element model (FEM) of the process was developed to better understand the experimental observations. The amount of surface expansion (plastic deformation) of the wire at the wire and substrate interface was extracted from the model and attributed to the degree of adhesion (bonding). The model was used to confirm the experimental observation of adhesion (bonding) with different surface finish.
\end{abstract}

Keywords: Pd coated wire (PCC), QFN Package, Stitch Bonding, Wire Bonding

\section{Introduction}

Thermosonic wire bonding is the most common interconnect method. It is widely used to connect the bond pads on a chip to the package the chip is attached to [1] and [2]. The most conventional wire is Au wire. However, recently Au wire is being replaced by $\mathrm{Cu}$ wire. $\mathrm{Cu}$ wire has better electrical and mechanical performance over Au wire, and is less expensive. One of the main disadvantages with $\mathrm{Cu}$ wire is its small process window with the stitch (also called second or wedge) bond. This is mainly due to $\mathrm{Cu}$ wire's rapid oxidation. [3]-[5]. To improve the stitch bond process with $\mathrm{Cu}$ wire, a Pd coating is introduced [6]-[9]. It is shown that Pd coated $\mathrm{Cu}$ wire (PCC) has a wider stitch bond process window than bare $\mathrm{Cu}$ wire. However its process window is still not comparable to that of $\mathrm{Au}$ and more studies are still required [10]-[13].

Currently quad flat no-lead (QFN) packages are widely used in the industry. They have enabled the industry to move to smaller package sizes and lower costs. However the stitch bond positions (lead fingers) on QFN packages are sensitive to ultrasonic vibration. Severe resonant oscillation has been observed upon applying ultrasonic energy which could result in unstable stitch bond processes and faulty bonds. To avoid such problem, processes without ultrasonic vibration are of interest. The ultrasonic energy can be replaced with other $\mathrm{x}-\mathrm{y}$ table motions. However, a process optimization and proper knowledge of the effect of parameters are required [14] and [15].

In the current study, the stitch bond process of PCC wire on QFN substrates was investigated. Stitch bonds are made with different capillary tool surface finishes and different process parameters and responses are extracted. A 3D finite element model of stitch bond processes was developed using ABAQUS software, and the experimental conditions were tested.

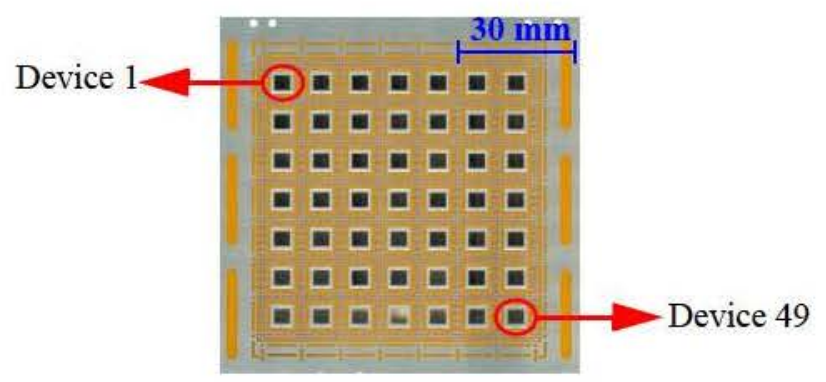

Fig. 1 Photograph of QFN package including 49 devices 


\section{Experimental}

A stitch bond process has been performed with $0.7 \mathrm{mil}(18$ $\mu \mathrm{m}$ ) diameter PCC wire on a $\mathrm{Au} / \mathrm{Ni} / \mathrm{Pd}$-plated $\mathrm{QFN}$ pre-plated (PPF) substrate. A photograph of a QFN substrate with 49 devices is shown in Fig. 1.

The bonding temperature and time were kept constant at 200 ${ }^{\circ} \mathrm{C}$ and $25 \mathrm{~ms}$, respectively. Contact velocity equal to $0.3 \mathrm{mils} /$ $\mathrm{ms}$ was used. To investigate the effect of the capillary surface, two capillaries with the same geometry, but different surface finishes were used. The capillaries have different surface roughness values: the rougher is called a granular finish and the smoother finish is the polished capillary. The granular capillary is an innovative capillary design from K\&S called CuPRA E3G. The smoother capillary is what is normally used for $\mathrm{Au}$ wire bonding. The geometry of the capillary tip is shown in Table I. The SEM images of different capillary surface finishes are shown in Fig. 2. To compare capillaries, process windows between no stick on lead (NSOL) and short tail are investigated.

The process parameters include bond force and low frequency table motion during bonding. To replace the ultrasonic vibration, a vibratory motion perpendicular to the wire with variable amplitude is used. This vibratory motion is generated by XY table motion, is often called table scrub. In the current work, 3 cycles of scrub at $100 \mathrm{~Hz}$ frequency are used. The stitch bond process consists of application of force, and scrub. Three levels of scrub amplitude $(1,3$, and $5 \mu \mathrm{m})$ are selected for comparison. Within each scrub amplitude level, force is varied from non-sticking on lead (NSOL) to $180 \mathrm{gf}$. The occurrence of NSOL and short tail are recorded for the tested process parameters, and process window is defined as the range of parameter settings that don't produce any NSOL or short tails. To have a robust wire bonding process with good yield and a low assist rate, the process window should be wide enough to accommodate normal variations from equipment and material.

In the next step, results of the process windows with different capillaries are compared with each other and 3 different process parameter settings which result in successful stitch bond with both capillaries are selected to do further study. The settings are selected to compare the capillaries at low, moderate, and high forces. The parameter settings are shown in Table II. Ten samples were bonded with each parameter setting and each capillary finish. Two responses were extracted from the stitch bonds made with different parameter settings; stitch pull force, and tail pull force. Stitch pull force is measured using a Dage 4000 series pull testing machine. Tail pull force is measured by using a built in function of the wire bonder

\section{Finite Element Model of Stitch Bond}

A finite element model of the stitch bond process was developed to understand the effect of process parameters on stitch bond formation. The dynamic explicit module of ABAQUS software was used for the simulation. For simplicity, no ther$\mathrm{mal}$ analysis is included. Instead of the whole geometry of the capillary, only a partial part of its tip is included. For the wire and the substrate, a piece of each of them was used instead of their whole geometry for simplicity. The model geometry is shown in Fig. 3. The normal force and scrub are included in the model. To include the effect of the roughness of different capillaries, the friction coefficient is measured for each case with other counterparts. To measure the friction coefficient, a custom designed test setup (discussed earlier in [11]) is used. The mechanical properties of PCC wire is extracted by using a custom designed uniaxial tensile test machine. It is assumed

Table I: Capillary geometry

\begin{tabular}{c|c|c|c|c|c}
\hline Feature & $\begin{array}{c}\text { Hole } \\
\text { Diameter } \\
{[\mu \mathrm{m}]}\end{array}$ & $\begin{array}{c}\text { Chamfer } \\
\text { Diameter } \\
{[\mu \mathrm{m}]}\end{array}$ & $\begin{array}{c}\text { Tip } \\
\text { Diameter } \\
{[\mu \mathrm{m}]}\end{array}$ & $\begin{array}{c}\text { Outer } \\
\text { Radius } \\
{[\mu \mathrm{m}]}\end{array}$ & $\begin{array}{c}\text { Face } \\
\text { Angle }\left[{ }^{\circ}\right]\end{array}$ \\
\hline Value & 23 & 27 & 70 & 5 & 11 \\
\hline
\end{tabular}
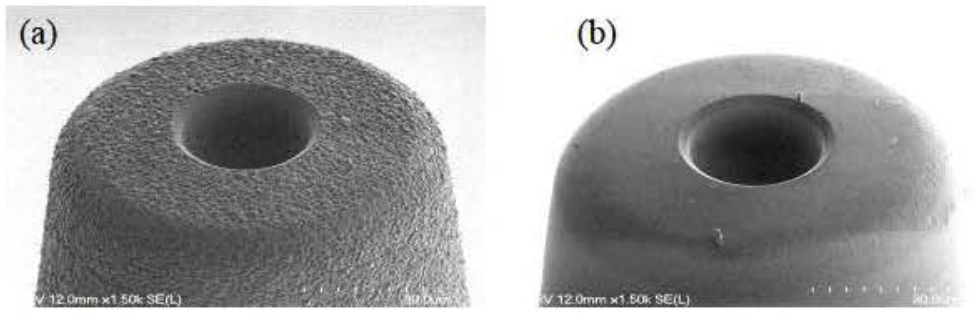

Fig. 2 SEM photograph of different capillaries (a) Granular and (b) Polished.

Table II: Stitch bond parameter settings for additional tests.

\begin{tabular}{c|c|c}
\hline setting \# & Force [g] & Scrub Amplitude [ $\mu \mathrm{m}]$ \\
\hline 1 & 40 & 5 \\
\hline 2 & 90 & 3 \\
\hline 3 & 140 & 3 \\
\hline
\end{tabular}




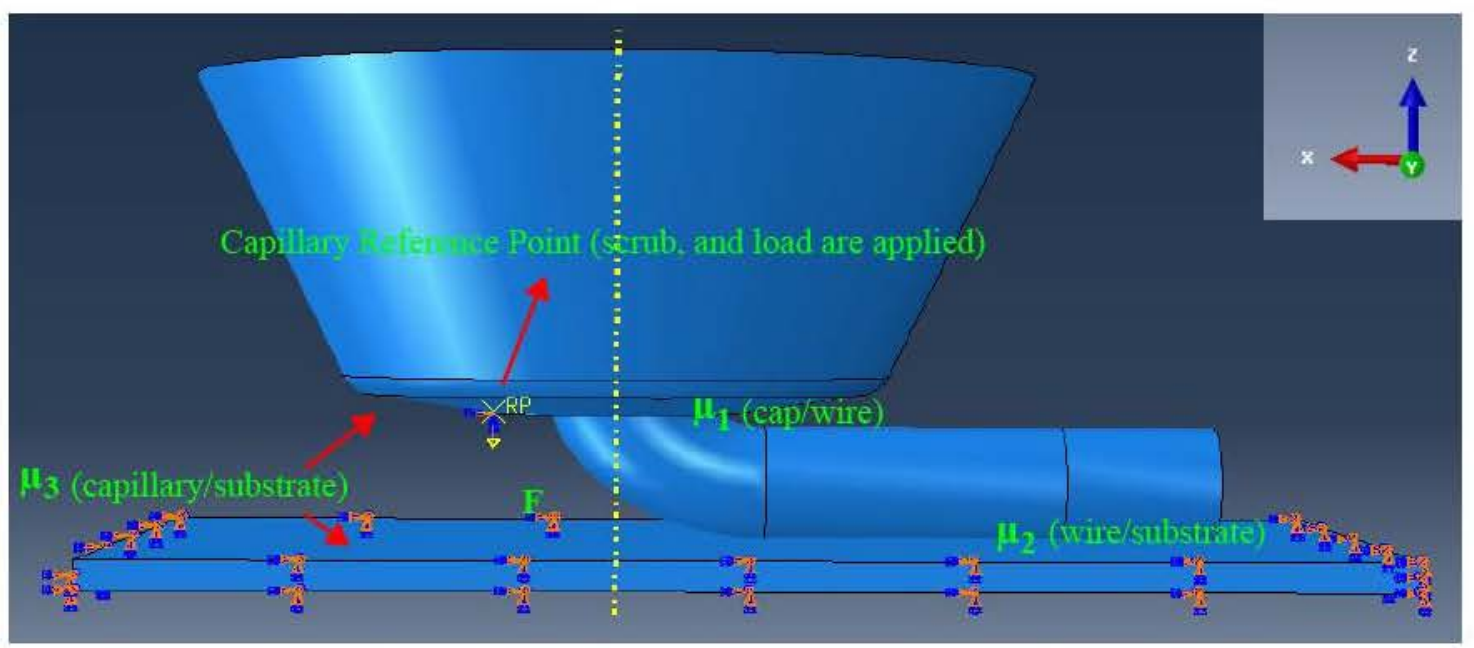

Fig. 3 Finite element model of stitch bond. Model geometry, load, boundary condition, and contacts are shown.

Table III: Model mechanical parameters

\begin{tabular}{|c|c|c|c|c|c|}
\hline Material \# & $\begin{array}{l}\text { Young modulus } \\
\text { [GPa] }\end{array}$ & Density $\left[\mathrm{g} / \mathrm{cm}^{3}\right]$ & $\begin{array}{c}\text { Poisson } \\
\text { ratio }\end{array}$ & $\begin{array}{c}\text { Yield Strength } \\
\text { [MPa] }\end{array}$ & $\begin{array}{c}\text { Tensile Strength } \\
\text { [MPa] }\end{array}$ \\
\hline $\begin{array}{l}\text { Wire (PCC) and } \\
\text { Substrate (QFN) }\end{array}$ & 100 & 8.9 & 0.35 & 140 & 265 \\
\hline Capillary & N/A (rigid) & N/A (rigid) & $\underset{\text { (rigid) }}{\text { N/A }}$ & N/A (rigid) & N/A (rigid) \\
\hline
\end{tabular}

Table IV: Model friction coefficients

\begin{tabular}{c|c|c|c|c}
\hline Material \# & Wire (PCC) & $\begin{array}{c}\text { Substrate } \\
(\mathbf{Q F N})\end{array}$ & $\begin{array}{c}\text { Capillary } \\
\text { (granular) }\end{array}$ & $\begin{array}{c}\text { Capillary } \\
\text { (polished) }\end{array}$ \\
\hline Wire (PCC) & N/A & 0.5 & 0.5 & 0.3 \\
\hline Substrate (QFN) & 0.5 & N/A & 0.5 & 0.3 \\
\hline Capillary (granular) & 0.5 & 0.5 & N/A & N/A \\
\hline Capillary (polished) & 0.3 & 0.3 & N/A & N/A \\
\hline
\end{tabular}

that $\mathrm{Cu}$ and PCC wires have similar mechanical properties and are different only in the surface. As a QFN substrate is a $\mathrm{Cu}$ bulk substrate coated with thin layers of $\mathrm{Au} / \mathrm{Ni} / \mathrm{Pd}$, the effect of coating layers is reflected in its friction coefficient, but not in the mechanical properties. The mechanical, and frictional properties of the model parts are shown in Tables III and IV, respectively.

The load is applied as a concentrated force to the capillary. As the capillary is rigid, it is defined by a reference point, and load is applied to that reference point. The wire is kept free in the model, but the substrate is subjected to a boundary condition which forbids any displacement or rotation for all of its sides except the top surface. The capillary is subjected to two boundary conditions, both applied to its reference point. First, all rotations are forbid $(\Delta U R x=\Delta U R y=\Delta U R z=0)$. Second, a periodic displacement perpendicular to the wire (y axis) is added (scrub). The Scrub cycle and frequency were fixed at 3 cycles, and $100 \mathrm{~Hz}$, same as the experimental.

\section{Results and Discussions}

\section{Process window with different capillary finishes}

The process window with different processes and capillary surface finishes are shown in Fig. 4. To improve the visualization, process responses are color coded. A blue color is assigned to NSOL, a white color is assigned to successful stitch bonds, and a red color is assigned to short tail occurrences. For a polished capillary, an NSOL condition tends to occur at the low force level and a short tail condition tend to occur at high scrub amplitude. By using a granular capillary finish, the short tail rate was decreased dramatically. Therefore, the process window is much larger than with a polished capillary.

The stitch bonds made with different capillaries and process parameters are shown in Fig. 5. The photos show that at a lower force and a higher scrub amplitude (setting \#1), stitch 

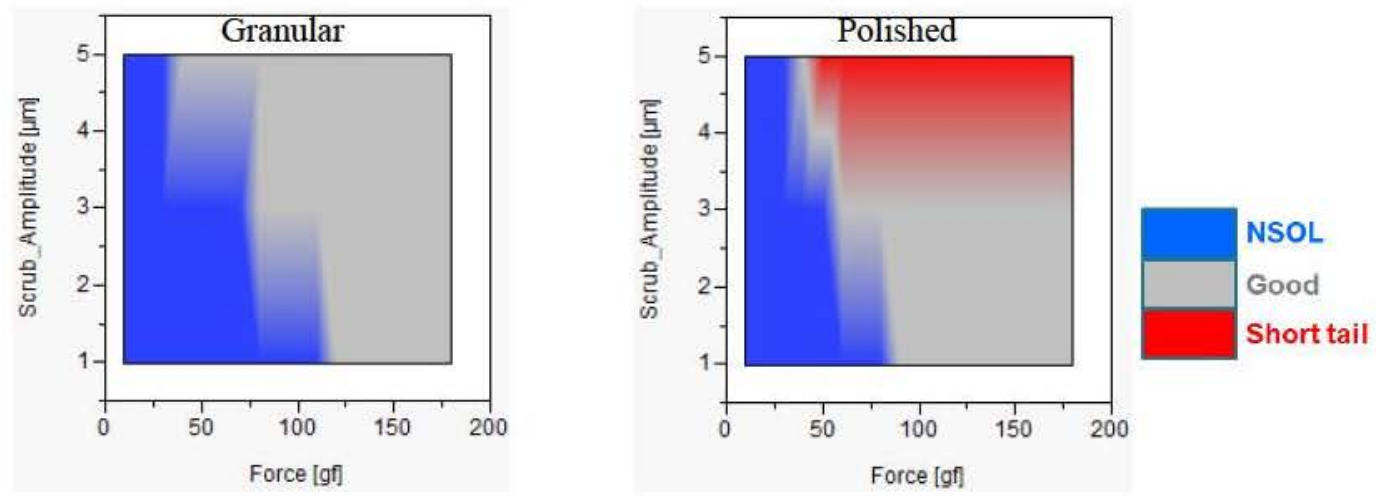

Fig. 4 Stitch bond process window (map) for different capillary surface finishes at different process parameters

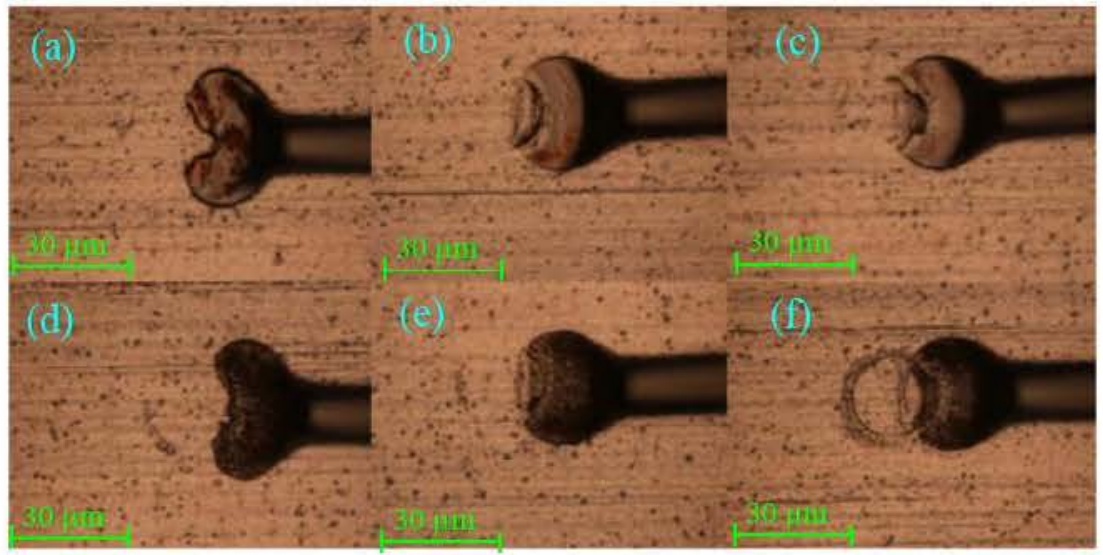

Fig. 5 Optical micrograph of stitch bonds made with different capillary finishes using process parameters (a) polished - se ting \#1 (b) polished - setting \#2 (c) polished - setting \#3 (d) granular - setting \#1 (e) granular - setting \#2 (f) granular setting \#3
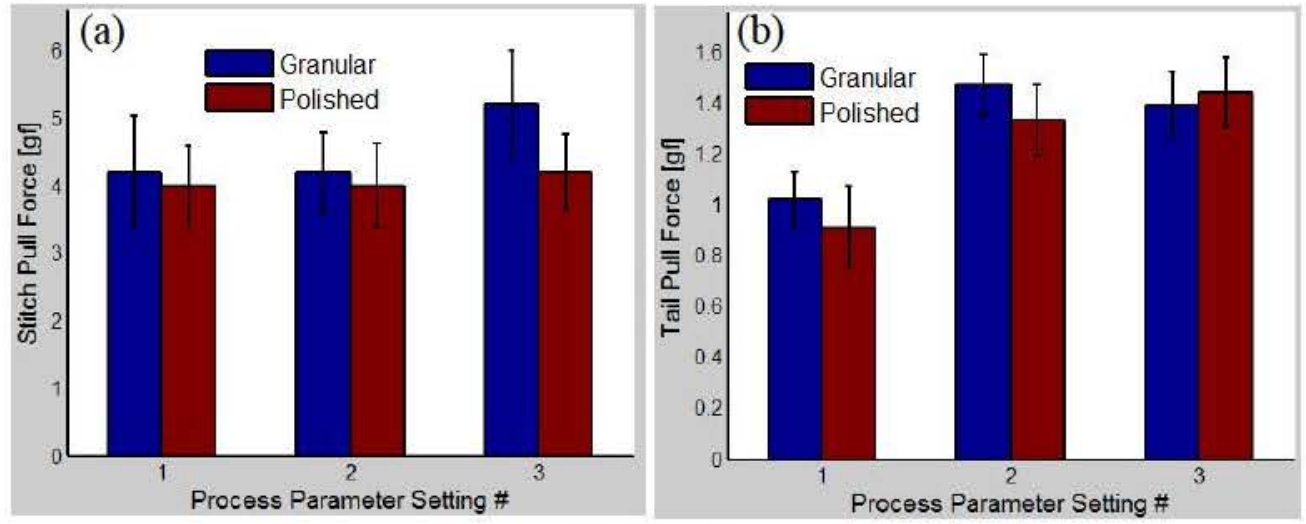

Fig. 6 Different parameter settings responses. (s) stitch pull force, and (b) tail pull force.

bonds are wider than other two settings. However the bonds made by settings \#2 and \#3 have a very similar stitch bond geometry. The photo shows that decreasing the granularity of the capillary finish decreases the quality of stitch bond with more 2nd bond peeling, especially for settings \#1 and \#3. The stitch pull force and tail pull force data for all three parameter settings for both capillary finishes are shown in Fig. 6. The bonds made with the granular capillary resulted in the highest stitch pull force in all 3 settings. This is in accordance with the observations of the process window testing. The granular capillary does not always result in highest tail pull force for all settings, as it showed higher tail strength in 2 out of 3 settings. These tail pull strengths are typically sufficient for a stable process.

\section{Finite Element Model of Stitch Bond}

Wire bonding is known as a solid - state process with no melting. The important factors influencing the bonding are diffusion and cold welding. The concept of cold welding has been extensively investigated by Bay et al [16]. It has been shown that for a successful cold weld, a certain amount of plastic deformation (area reduction) at the interface is required. This plastic deformation threshold might vary if different bonding pairs are used [16] and [17]. Assuming the similarity between stitch bond and cold weld processes, plastic deformation at the back side of the wire is selected to compare the bonding (adhesion) for different cases. 

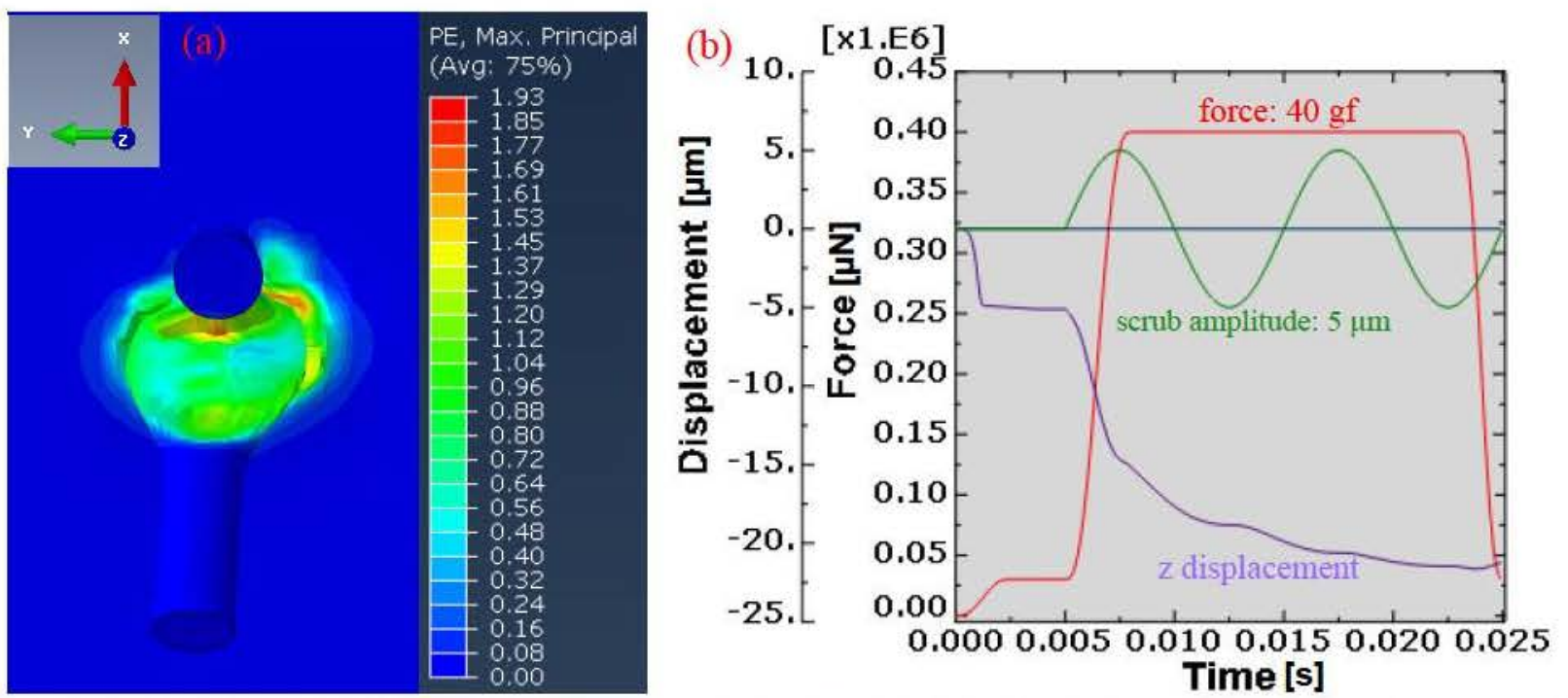

Fig. 7 Model results of bond formed with process parameters for setting \#1. (a) top view of deformed wire, and (b) three dimensional movement of capillary and force profile
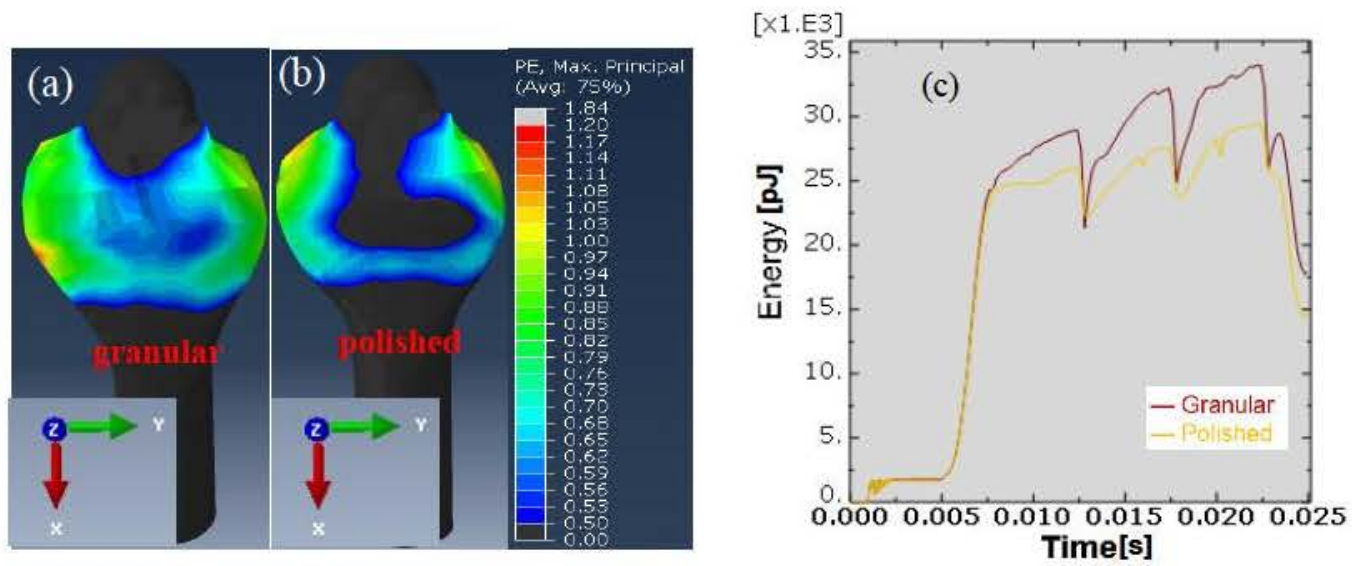

Fig. 8 Plastic distribution at backside of wire for bonds made by setting \#1 condition (a) granular and (b) polished. (c) Variation of strain energy during simulation for both capillaries. Larger deformation (expansion) of wire surface increases the strain energy.

The top view of the deformed wire and three dimensional movement of the capillary reference point for process parameters \#1 and granular capillary finish are shown in Fig. 7. To compare both capillary finishes, the plastic strain distribution at the backside of the wire, and strain energy, are plotted for each case in Fig. 8. To make the comparison possible, arbitrarily the amount of plastic strain equal to 0.5 is selected as a bonding threshold. So, for each case, contour plots of plastic strain greater than 0.5 were plotted at the backside of the wire. In Figs. 8 (a) to (b), it is qualitatively shown that a polished capillary will result in less bonded area. This is in accordance with experimental observations. However, it does not give an exact number for the bond strength. The other extracted output is the amount of the strain energy for the model. It is shown for both capillaries in Fig. 8. Fig. 8 illustrates that the granular capillary results in a higher strain energy than the polished capillary. This might correlate with the earlier experimental and numerical observations. In the future, it is planned to extract responses which could be represented by a final number. It is believed that such responses will enhance the experiment-model correlation.

\section{Conclusions}

- It is shown that using capillaries with a rougher (more granular) surface enhances the process window of the stitch bond process of PCC wire on QFN substrates. Capillaries such as the K\&S CuPRA E3G design enables better 2 nd bond performance.

- Granular capillaries typically result in a higher stitch pull force than polished capillary surface finishes. Such capillaries also typically result in an adequate tail pull force.

- Increasing scrub amplitude and force tends to increase the chance of a successful stitch bond formation. Excessive usage of these parameters tends to increase the chance of a short tail occurrence, especially with the polished capillary.

- A finite element model of a stitch bond process is developed. If bonding/adhesion is attributed to the plastic strain at the backside of the wire, the model provides a qualitative correlation with the experiments utilizing capillaries with different surface finishes. 


\section{References}

[1] Harman, George G. Wire bonding in microelectronics: materials, processes, reliability, and yield. Vol. 21. New York: McGraw-Hill, 1997, pp. 1-10

[2] Prasad, Shankara K. Advanced wirebond interconnection technology. Springer, 2004, pp. 1-14

[3] Rezvani, A., A. Shah, M. Mayer, Y. Zhou, and J. T. Moon. "Role of impact ultrasound on bond strength and Al pad splash in $\mathrm{Cu}$ wire bonding." Microelectronics Reliability (2013).

[4] Rezvani, A., M. Mayer, A. Shah, N. Zhou, S. J. Hong, and J. T. Moon. "Free-air ball formation and deformability with Pd coated $\mathrm{Cu}$ wire." In Electronic Components and Technology Conference (ECTC), 2011 IEEE 61st, pp. 1516-1522. IEEE, 2011.

[5] Shah, A., A. Rezvani, M. Mayer, Y. Zhou, J. Persic, and J. T. Moon. "Reduction of ultrasonic pad stress and aluminum splash in copper ball bonding." Microelectronics Reliability 51, no. 1 (2011): 67-74.

[6] Uno, Tomohiro, Shinichi Terashima, and Takashi Yamada. "Surface-enhanced copper bonding wire for LSI." In Electronic Components and Technology Conference, 2009. ECTC 2009. 59th, pp. 1486-1495. IEEE, 2009.

[7] Uno, Tomohiro. "Enhancing bondability with coated copper bonding wire." Microelectronics Reliability 51, no. 1 (2011): 88-96.

[8] Uno, Tomohiro. "Bond reliability under humid environment for coated copper wire and bare copper wire." Microelectronics Reliability 51, no. 1 (2011): 148-156.

[9] Uno, Tomohiro, Keiichi Kimura, and Takashi Yamada. "Surface-enhanced copper bonding wire for LSI and its bond reliability under humid environment." In Microelectronics and Packaging Conference, 2009. EMPC 2009. European, pp. 1-10. IEEE, 2009.
[10] Liu, Peisheng, Liangyu Tong, Jinlan Wang, Lei Shi, and Hao Tang. "Challenges and developments of copper wire bonding technology." Microelectronics Reliability 52, no. 6 (2012): 1092-1098.

[11] Rezvani, A., C. Nan, M. Mayer, and I. Qin. "The role of friction coefficient on the stitch bondability in Pd coated $\mathrm{Cu}$ and bare $\mathrm{Cu}$ wire bonding." In Electronic Components and Technology Conference (ECTC), 2012 IEEE 62nd, pp. 2150-2156. IEEE, 2012.

[12] Singh, Inderjit, Ivy Qin, Hui Xu, Cuong Huynh, Shin Low, Horst Clauberg, Bob Chylak, and Viola L. Acoff. "Pd-coated $\mathrm{Cu}$ wire bonding technology: Chip design, process optimization, production qualification and reliability test for high reliability semiconductor devices." In Electronic Components and Technology Conference (ECTC), 2012 IEEE 62nd, pp. 1089-1096. IEEE, 2012.

[13] Schneider-Ramelow, Martin, Ute Geißler, Stefan Schmitz, Wolfgang Grübl, and Bernhard Schuch. "Development and Status of Cu Ball/Wedge Bonding in 2012." Journal of electronic materials 42 , no. 3 (2013): 558-595.

[14] Wei, Tan Boo, and Ken Niu. "Study of Factors Contributing to Robust Copper Wire Bond on QFN." ECS Transactions 44, no. 1 (2012): 985-989.

[15] Lin, Yi-Chieh, Yu-Chih Lin, Tzyy-Sheng Horng, LihTyng Hwang, Chi-Tsung Chiu, and Chih-Pin Hung. "Low cost QFN package design for millimeter-wave applications." In Electronic Components and Technology Conference (ECTC), 2012 IEEE 62nd, pp. 915-919. IEEE, 2012.

[16] Bay, Niels. "Mechanisms producing metallic bonds in cold welding." WELDING J. 62, no. 5 (1983): 137.

[17] Li, Long, Kotobu Nagai, and Fuxing Yin. "Progress in cold roll bonding of metals." Science and Technology of Advanced Materials 9, no. 2 (2008): 023001. 